\title{
Automatic and Portable Mapping of Data Parallel Programs to OpenCL for GPU-Based Heterogeneous Systems
}

\author{
ZHENG WANG, Lancaster University \\ DOMINIK GREWE and MICHAEL F. P. O'BOYLE, University of Edinburgh
}

General-purpose GPU-based systems are highly attractive, as they give potentially massive performance at little cost. Realizing such potential is challenging due to the complexity of programming. This article presents a compiler-based approach to automatically generate optimized OpenCL code from data parallel OpenMP programs for GPUs. A key feature of our scheme is that it leverages existing transformations, especially data transformations, to improve performance on GPU architectures and uses automatic machine learning to build a predictive model to determine if it is worthwhile running the OpenCL code on the GPU or OpenMP code on the multicore host. We applied our approach to the entire NAS parallel benchmark suite and evaluated it on distinct GPU-based systems. We achieved average (up to) speedups of $4.51 \times$ and $4.20 \times(143 \times$ and $67 \times$ ) on Core i7/NVIDIA GeForce GTX580 and Core i7/AMD Radeon 7970 platforms, respectively, over a sequential baseline. Our approach achieves, on average, greater than $10 \times$ speedups over two state-of-the-art automatic GPU code generators.

Categories and Subject Descriptors: D.3.4 [Programming Languages]: Processors—Compilers

General Terms: Experimentation, Languages, Measurement, Performance

Additional Key Words and Phrases: GPU, OpenCL, Machine-learning mapping

ACM Reference Format:

Zheng Wang, Dominik Grewe, and Michael F. P. O'Boyle. 2014. Automatic and portable mapping of data parallel programs to OpenCL for GPU-based heterogeneous systems. ACM Trans. Architec. Code Optim. 11, 4, Article 42 (December 2014), 26 pages.

DOI: http://dx.doi.org/10.1145/2677036

\section{INTRODUCTION}

Heterogeneous systems consisting of a host multicore and general-purpose GPU are highly attractive, as they give potentially massive performance at little cost. Realizing such potential, however, is challenging due to the complexity of programming. Users typically have to identify potential sections of their code suitable for SIMD style parallelization and rewrite them in an architecture-specific language. To achieve good performance, significant rewriting may be needed to fit the GPU programming model and to amortize the cost of communicating to a separate device with a distinct address

Extension of Conference Paper: A preliminary version of this article entitled "Portable Mapping of Data Parallel Programs to OpenCL for Heterogeneous Systems” by D. Grewe, Z. Wang, and M. O’Boyle appeared in International Symposium on Code Generation and Optimization (CGO)[Grewe et al. 2013a].

Authors' addresses: Z. Wang, School of Computing and Communications, Lancaster University; email: z.wang@lancaster.ac.uk; D. Grewe, Google London, 76 Buckingham Palace Road, London SW1W 9TQ, United Kingdom; email: dominik.grewe@googlemail.com; M. F. P. O’Boyle, School of Informatics, University of Edinburgh; email: mob@inf.ed.ac.uk.

Permission to make digital or hard copies of part or all of this work for personal or classroom use is granted without fee provided that copies are not made or distributed for profit or commercial advantage and that copies show this notice on the first page or initial screen of a display along with the full citation. Copyrights for components of this work owned by others than ACM must be honored. Abstracting with credit is permitted. To copy otherwise, to republish, to post on servers, to redistribute to lists, or to use any component of this work in other works requires prior specific permission and/or a fee. Permissions may be requested from Publications Dept., ACM, Inc., 2 Penn Plaza, Suite 701, New York, NY 10121-0701 USA, fax +1 (212) 869-0481, or permissions@acm.org.

(c) 2014 ACM 1544-3566/2014/12-ART42 $\$ 15.00$

DOI: http://dx.doi.org/10.1145/2677036 
space. Such programming complexity is a barrier to greater adoption of GPU-based heterogeneous systems.

OpenCL is emerging as a standard for heterogeneous computing. It provides portability, which allows the same code to be executed across a variety of processors, including multicore CPUs and GPUs. By exposing fine-grained parallelism, carefully written OpenCL (or CUDA) programs can achieve good performance across parallel processor architectures [Stratton et al. 2010].

However, there are many legacy programs written with shared memory programming languages such as OpenMP [Lee et al. 2009]. To benefit from heterogeneous performance, it will require considerable development efforts to rewrite those programs with OpenCL, and the process can be prone to error [Lee and Eigenmann 2010]. This work aims to provide a simple upgrade path for targeting OpenMP programs on heterogeneous platforms. We achieve this by developing a compiler-based approach that automatically generates optimized OpenCL from a subset of OpenMP and using machine learning to determine the best-performing processor on a CPU-GPU mixed system. This allows the user to run the same data parallel program written in OpenMP, which has been fully tested, with no modifications while benefiting automatically from heterogeneous performance.

The first effort in this direction is proposed in Lee et al. [2009]. Here, the OpenMPC compiler generates CUDA code from OpenMP programs. While promising, there are two significant shortcomings with this approach. First, OpenMPC does not apply data transformations. As shown in this article, data transformation is crucial to achieve good performance on GPUs (Sections 4 and 7.4). Second, the programs are always executed on GPUs. Although GPUs may deliver improved performance, they are not always superior to CPUs [Bordawekar et al. 2010; Lee et al. 2010a]. A technique for determining when GPU execution is beneficial is needed. This article addresses both of these issues, and when evaluated on the full NAS parallel benchmarks, our technique outperforms OpenMPC by a factor of 10 .

Our work examines performance portability across heterogeneous platforms, considering the trade-offs in heterogeneity-it may be better to run the program as OpenMP on a multicore rather than as OpenCL on the GPU. It uses OpenCL as a target language and is applied to the entire NAS parallel benchmark suite on different GPU-based systems from different vendors, using automatic machine learning techniques to predict the best components to use in a heterogeneous system. We generate high-quality OpenCL code achieving up to $202 \times$ speedup over sequential $\mathrm{C}$ for the ep benchmark on AMD Radeon automatically. This article's technical contributions can be summarized as follows. It is the first to:

—use machine learning to decide between different implementation languages on heterogeneous platforms (Section 5),

- use machine learning to automatically build cost-based models for dynamic array index reordering for GPUs (Section 4), and

-automatically translate and map all NAS parallel benchmarks onto GPUs; some of benchmarks are up to 3,600 lines long with 66 kernels - a nontrivial task (Section 7).

A key feature of our scheme is that it uses machine learning to build predictive models to automatically determine if it is worthwhile running the code on the GPU or the multicore host. Furthermore, it can adapt this model to different GPU architectures and generations. We also show that data transformations can be used to significantly improve performance on GPU architectures. This means that the user can use the same OpenMP code on different platforms with the compiler determining the best place for code to run and optimize it accordingly. 


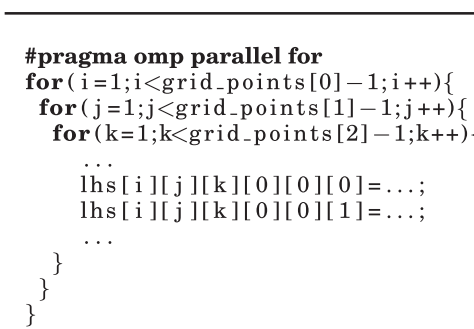

(a) Original OpenMP

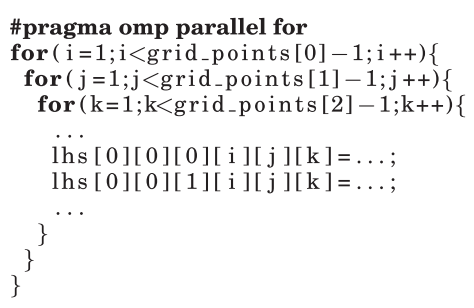

(d) Transformed OpenMP

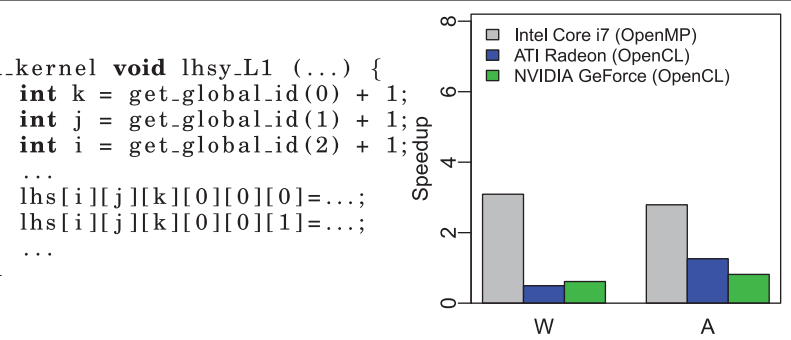

(b) Nonoptimized OpenCL

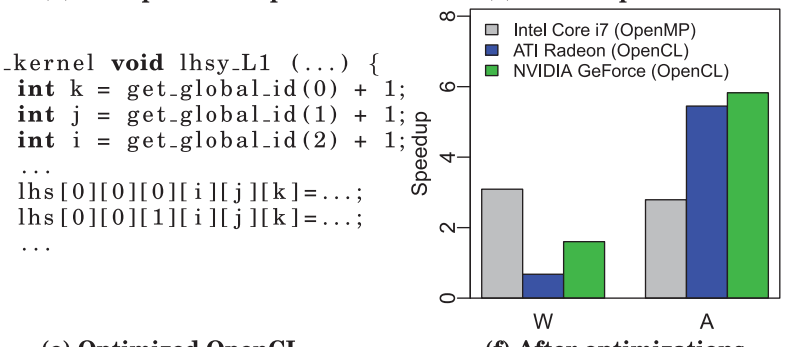

(e) Optimized OpenCL

Fig. 1. Simplified example of generating OpenCL code from OpenMP code. The top left code snippet (a) is taken from bt. The corresponding OpenCL code (b) delivers poor performance on both GPUs (c). After applying data transformation to the OpenMP code (d), we obtain the new OpenCL code shown in (e). The performance of both GPUs improves significantly, but only for large inputs can they outperform the CPU (f).

\section{MOTIVATION}

With the massive interest in GPUs, it is important to know that GPUs are not always the most suitable device for scientific kernels. This section provides a simple example demonstrating that the appropriateness of GPU computation depends on the original program, data size, and the transformations available.

Consider the OpenMP fragment in Figure 1(a) from the NAS parallel benchmark bt, a benchmark containing more than 60 parallel loops potentially suitable for offloading to a GPU. This program was executed with two different programs input: a small input, $\mathrm{W}$, and a large input, A. Using our basic OpenMP to OpenCL translator yields the code shown in Figure 1(b). The parallel loop has been translated into a kernel where each of the loops is parallelized forming a 3D parallel work-item space (i.e., ND-Range), each point of which is accessed through a call to get_global_id for dimensions 0,1 , and 2 .

If executed on a GPU with the W input size, however, this code gives disappointing performance when compared to executing the code on a multicore as shown in Figure 1(c). This is largely due to a relatively small work to be performed on the GPU, where a high percentage of coalesced memory access is required to achieve good performance (see Table IV in Section 7.6). If we execute the same code with a larger input size A, the GPU performance improves but is still less than the performance achieved on the multicore, primarily because of the memory access pattern of the kernel, which does not allow for memory coalescing on the GPU. This can be changed by performing global index reordering (see Section 4.1) as shown in Figure 1(d), transforming the data layout of array lhs. This gives the new OpenCL program shown in Figure 1(e). Here, the most rapidly varying indexes of the array correspond to the tile IDs giving coalesced memory accesses. As can be seen later in Table IV, this improves the percentage of coalesce memory access (feature F2 in Table IV) from 0 to 0.78 and 0.999 for input sizes W and A, respectively. In Figure 1(f), we see that the resulting performance of the GPU code improves substantially for data size W. If this transformed code is executed 


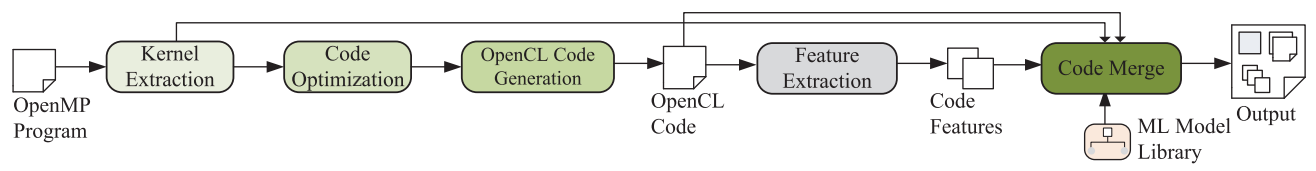

Fig. 2. Overview of our compilation framework.

with a larger data size A, the GPU performance further improves, with both GPUs now outperforming the multicore OpenMP implementation.

This example shows that the translated OpenCL code can give better performance than the original OpenMP code depending on the data size and transformations available. As described in Section 6, this decision varies from program to program and across different platforms and data sizes, and depends on a number of factors (see Figures 13 and 14). What we would like is a system that learns when to use the GPU, changing its decision based on the availability of underlying optimizations such as data layout transformations.

\section{OVERALL SCHEME}

Our compiler automatically translates OpenMP programs to OpenCL-based code, performing loop and array layout optimizations along the way. It generates multiversioned code for each parallel loop: the original OpenMP parallel loop and an optimized OpenCL kernel alternative. At runtime, a machine learning (ML)-based predictive model decides which version to use for execution. Our prototype compiler is implemented using Clang (v3.0) and LLVM (v3.0) [LLVM 2013].

\subsection{Compile Time}

Figure 2 gives an overview of our approach. The OpenMP program is read in and parallel loops are optimized and translated to OpenCL kernels. The generated kernels are passed to a feature extraction phase that collects characteristics or features from the Abstract Syntax Tree of the generated OpenCL code. These features are later used by the ML model to select whether the OpenMP loop or OpenCL kernel version is best (Section 4). The features, together with the generated OpenCL code, the original OpenMP code, and a ML predictor built offline (Section 5) are merged into a single output program source.

\subsection{Runtime}

At execution time, the program first updates the parameterized feature values based on the runtime values of parameters and passes the instantiated feature values to the ML model. The built-in model then predicts where to run the program and to pick either the OpenMP version for a multicore CPU or the OpenCL version for a GPU. Evaluating the model at runtime involves on the order of tens of operations and is thus negligible. This is the high-level overview of the compilation framework. The next sections describe each of the stages in more detail.

\section{CODE GENERATION AND OPTIMIZATION}

Table I lists the OpenMP directives supported by our implementation.

Work-Sharing Constructs. Our compiler converts OpenMP parallel loops-that is, loops that are annotated with omp for or omp for reduction-into OpenCL kernels. Other parallel OpenMP directives associated with task parallelism are not currently supported. A two-stage algorithm [AMD 2013] is used to translate parallel reduction loops. 
Table I. Supported OpenMP Directives

\begin{tabular}{|l|llll|}
\hline Parallel Constructs etc. & parallel & for & for reduction & \\
\hline Data Attributes & default & shared & private & first_private \\
& last_private & threadprivate & copyin & \\
\hline Other Constructs & barrier & atomic & critical & master \\
& single & flush & & \\
\hline
\end{tabular}

Data Attributes. In OpenMP, variables may have additional type information specified by directives: default, shared, private, first_private, last_private, copyin, and threadprivate. Our framework uses these directives to map data onto the GPU memory space. Each variable with the share or default directive will be translated into an OpenCL global variable shared by all OpenCL threads. Variables declared as private and threadprivate are translated such that there is a private copy for each OpenCL work item; no memory transfer between the GPU and the CPU is needed. For each variable specified as copyin or first_private, we create a private copy for each OpenCL work item but initialize each copy using explicit memory transfers before its first use. Similarly, we create a private copy of a last_private variable, and the original variable is updated by the GPU thread that executes the last work item.

Other Constructs. Our implementation also supports a number of synchronization and thread constructs. Structured blocks identified with master, single, and critical directives are executed by one thread on the host multicore. barrier is implemented by calling the OpenCL clFinish API to synchronize all OpenCL threads. An atomic operation is translated into the corresponding OpenCL atomic function according to the type of the operand (variable). The current level of atomic support can be easily extended to support part of the C11 atomics, such as atomic load and stores. To fully support the C11 atomic standard is our future work. Finally, flush is implemented using the OpenCL barrier (CLK_LOCAL_MEM_FENCE | CLK_GLOBAL_MEM_FENCE) API.

\subsection{OpenCL Code Optimization}

Our compiler performs a number of optimizations to improve the performance of the OpenCL code on the GPU. ${ }^{1}$ The optimizations implemented in our compiler are applied in the following order.

Loop Interchange. High memory bandwidth on GPUs can only be achieved when memory accesses are coalesced-that is, adjacent threads access adjacent memory locations in the GPU off-chip memory. Our framework applies loop interchange to place outermost those iterators that occur most frequently in the innermost array subscripts. We use the LLVM DependenceAnalysis pass to detect to which level the nested loop can be interchanged.

Global Index Reordering. Global index reordering is the data structure equivalent of loop reordering. Indexes of an array are permuted to fit an optimization purpose. This transformation is necessary when loop interchange cannot provide memory coalescing. This can be represented as $\left[s_{1}, s_{2}, \ldots, s_{n}\right] \mapsto\left[s_{x_{1}}, s_{x_{2}}, \ldots, s_{x_{n}}\right]$, where each $x_{k} \in 1, \ldots, n \wedge \forall i, j,\left(x_{i}=x_{j}\right) \Rightarrow(i=j)$. In our case, we wish to place outermost those indexes that appear as OpenCL work-item indexes. Given that there are a maximum of three parallel work-item indexes, $t_{1}, t_{2}, t_{3}$, then for any one access we wish $s_{x_{n}}=t_{1}, s_{x_{n-1}}=t_{2}, s_{x_{n-2}}=t_{3}$. An example of this transformation was shown in Figure 1 : $[i, j, k, 0,0,0] \mapsto[0,0,0, i, j, k]$.

1The LLVM “-O3" optimization stack also provides other types of optimizations that primarily perform on the LLVM IR level. Integrating this into our source to source OpenCL code generator is the future work. 
After index reordering, our compiler renames the function prototype where input array arguments will be updated accordingly and changes the code where this function is called. This removes the need for additional array copying (in and out) to comply with the calling context. Global index reordering requires global alias analysis to ensure correctness. To do so, we run the code through the "-basicaa" and "-steens-aa" passes provided by LLVM, where the later pass implements the well-known Steensgaard's pointer analysis [Steensgaard 1996]. Note that our current implementation only applies global index reordering to statically allocated arrays.

Dynamic Index Reordering. In conjunction with loop interchange global index, reordering is often sufficient to achieve memory coalescing. However, in some cases, there is no clear best global data layout, such as when different loops in a program require different layouts to achieve memory coalescing. We then consider dynamic index reordering [Che et al. 2011].

Before entering a code region containing loops that prefer a certain index order for an array $X$ (different from the global one), a reordered copy of the array, $X^{\prime}$, is created. During the copy process, the data gets reordered as follows. Given a preferred access pattern $\left[s_{x_{1}}, s_{x_{2}}, \ldots, s_{x_{n}}\right]$, we copy $X$ to $X^{\prime}$ such that $X^{\prime}\left[s_{x_{1}}, s_{x_{2}}, \ldots, s_{x_{n}}\right]=X\left[s_{1}, s_{2}, \ldots, s_{n}\right]$. Within the code section, all references to $X$ are redirected to $X$ and the indexes are reordered appropriately. At the end of the region, the data gets copied back to the original array.

Dynamic index reordering for GPU computing can often be prohibitive. The transformation should only be applied if the benefits of data coalescing outweigh the costs of data reordering. To be used in a compiler setting, we therefore need a mechanism to automatically determine when this transformation should be applied. Section 5.4 describes an ML-based cost model that solves this problem.

Memory Load Reordering. In the original OpenMP programs, accesses to read-only buffers might be reordered to form a sequence of consecutive load operations that can be vectorized. Our compiler automatically detects those accesses and replaces scalar load operations with an OpenCL vector load operation. Our current implementation simply groups consecutive load and store operations together and replace them with an OpenCL vloadn or vstoren $(n=2,4,8,16)$ operation. This is a standard optimization technique that has been used in prior work [Eichenberger et al. 2004; Yang et al. 2010].

Register Promotion. On many occasions, a scalar variable (or array) stored in the global memory space is accessed multiple times by a single OpenCL kernel. To reduce global memory latencies, our tool automatically creates a private register object for such a variable. It generates code to load the data from the global memory to the private register copy (and write back to the global memory from the register after the last store operation). Doing so can eliminate redundant global memory loads and stores and thus improves performance. Depending on the implementation, the backend OpenCL compiler might also perform register promotion optimization [Lu and Cooper 1997; Chakrabarti et al. 2012] on the generated code.

Prefetching and Local Memory Optimization. For read-only buffers (that are used by multiple GPU threads) identified by our compiler, we generate code to prefetch the data to the local memory. Exploiting local memory generally reduces memory latencies for GPU kernels [Lee et al. 2010b].

Host-Device Communication. For each array that is used by both the host and the GPU, we manage two copies: one on the host memory and the other on the GPU memory. Our runtime records the status of each variable and checks whether the copy on a device memory space is valid or not. No memory transfer is needed as long as 


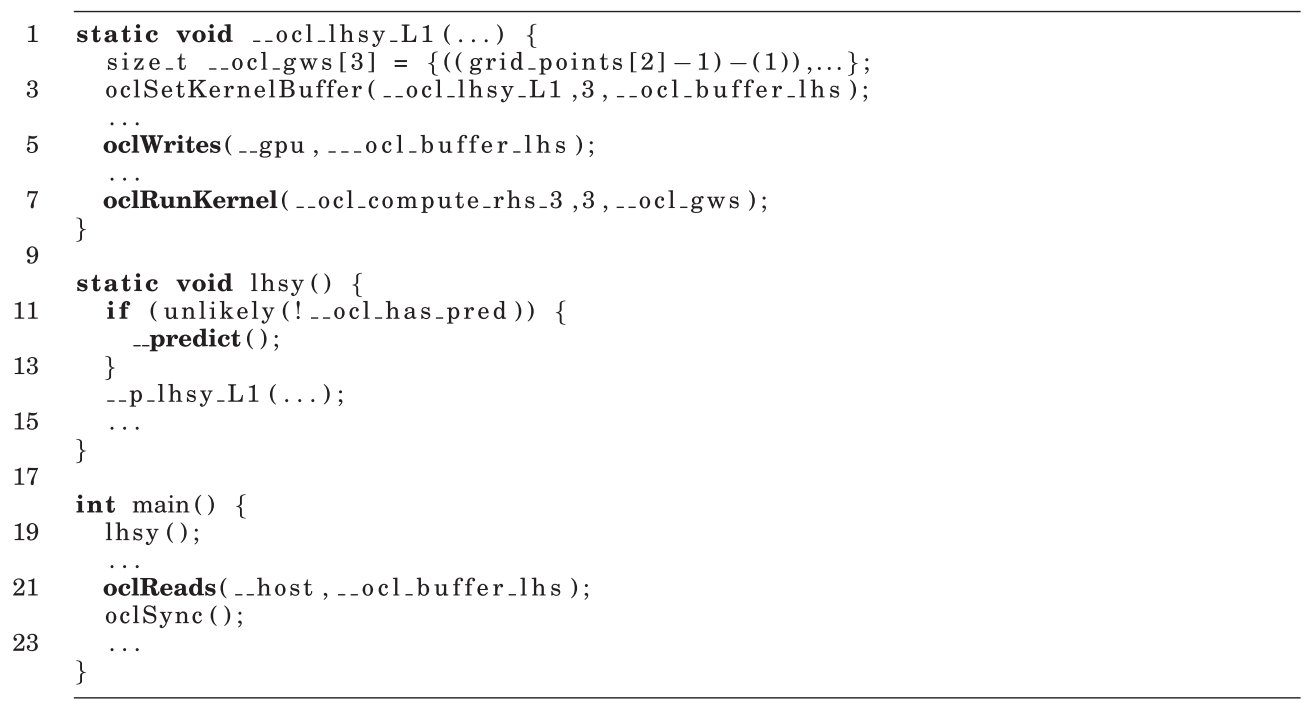

Fig. 3. The generated host code of the loop shown in Figure 1(a).

the copy in the target memory space is valid. Our current implementation uses a conservative approach: if an element of an array has been updated, the entire array needs to be synchronized before it can be used by threads running on a different device. There are advanced techniques available for host-device communication optimizations [Jablin et al. 2012; Margiolas and O’Boyle 2014], which are orthogonal to our approach.

\subsection{Host Code Generation}

For each parallel loop, we outline the loop body and generate two versions for it: an OpenCL and an OpenMP version. The original loop body is replaced with a function pointer that points to either the OpenCL or OpenMP version of the loop. For example, the OpenCL version of the original loop shown in Figure 1(a) is translated to _-_ocl_lhsy_L1 as shown in Figure 3. We try to generate as many work items as possible to utilize the GPU. Each iteration of the nested parallel loops is translated into a OpenCL work item. The dimension of the ND-range is determined by the number of nested loops to be parallelized (up to three dimensions due to the restriction of OpenCL). In Figure 3, the OpenCL work item indexes are calculated at line 2, and are then sent to the OpenCL runtime at line 7. The original loop body is replaced with a function pointer at line 14. Each generated code has a prediction function, _- predict, that decides which device to use to run the program. This is done by setting the function pointer of each loop to the corresponding code version. Currently, we use a single program version for all parallel loops and do not use both versions interleaved at runtime. Finally, host-device communication is managed by our communication library, where read and write operations to OpenCL buffers are identified through the oclReads and oclWirtes functions, respectively (line 5). The oclWirtes function keeps track of which device has recently updated the input OpenCL buffer. This information will be used to determine whether a data transfer is needed at runtime.

\section{PREDICTING THE MAPPING}

A crucial part of our approach is to automatically determine the best computing device for the input program, whether it should be run on the multicore host or translated into OpenCL and executed on the GPU. Our approach is to generate the OpenCL-based 


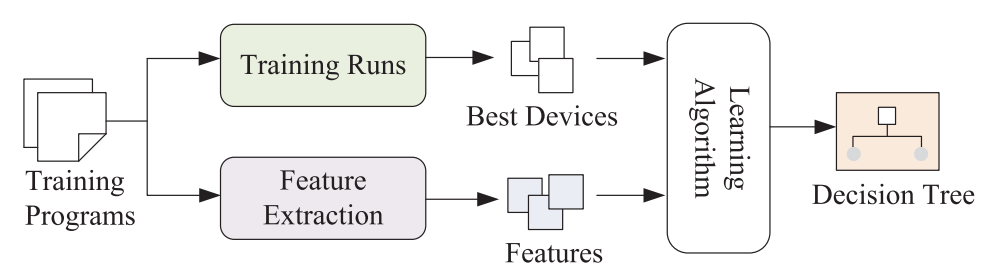

Fig. 4. The process of training a decision tree classifier.

Table II. List of Code Features

(a) Individual code features

\begin{tabular}{ll}
\hline & Raw Code Features \\
\hline comp & Number of compute operations \\
mem & Number of accesses to global memory \\
localmem & Number of accesses to local memory \\
coalesced & Number of coalesced memory accesses \\
transfer & Amount of data transfers \\
avgws & Average number of work items per kernel \\
\hline
\end{tabular}

(b) Combinations of raw features

\begin{tabular}{lc}
\hline & Combined Code Features \\
\hline F1: transfer/(comp+mem) & Communication-computation ratio \\
F2: coalesced/mem & $\begin{array}{l}\text { Percentage of coalesced memory accesses } \\
\text { F3: (localmem/mem) } \times \text { avgws }\end{array}$ \\
$\begin{array}{l}\text { Ratio of local to global memory accesses } \\
\times \text { average number of work items per kernel }\end{array}$ \\
F4: comp/mem & Computation-memory ratio \\
\hline
\end{tabular}

code and then use an ML model to see if this is profitable to run on a GPU. If it is not profitable, we fall back to the original OpenMP code. As this decision will vary greatly depending on GPU architectures and the maturity of the OpenCL runtime, we wish to build a portable model that can adapt to the change of the architecture and runtime.

We prefer to avoid any additional profiling runs or exhaustive search over different datasets, so our decision is based on static compiler analysis of the abstract syntax tree and runtime parameters. The static analysis characterizes a kernel as a fixed vector of real values, or features.

\subsection{Training the Predictor}

Figure 4 shows the process of training the predictor-in our case, a decision tree classifier. This involves the collection of a set of training data that is used to fit the model to the problem at hand. In this work, we use a set of programs that are each executed on the CPU and the GPU to determine the best device in each case. We also extract code features for each program as described in the following section. The features, together with the best device for each program from the training data, are used to build the model. Since training is only performed once at the factory, it is a one-off cost. In our case, the overall training process takes less than a day on a single machine.

\subsection{Code Features}

Our predictor is based on code features (Table IIa). These are selected by the compiler writer and summarize what are thought to be significant costs in determining the mapping. At compile time, we analyze the OpenCL code and extract information about the number and type of operations. We developed a Clang-based tool to extract 
those features from the abstract syntax tree of the code. Double precision floating point operations are given a higher weight $(4 \times)$ than single precision operations, as they are expensive on many GPU architectures. Obviously, this weight is highly platform dependent, but this can be estimated by using microbenchmarks to test how much extract time is needed for performing the same number of double floating point operations versus single floating operations. Using an analytical model similar to Sim et al. [2012], we also analyze the memory access pattern to determine whether or not an access to global memory is coalesced. A potential feature is the amount of control flow in an application. Although this feature can have an impact on performance on the GPU, it was not relevant for the benchmarks that we considered. It is thus not included in our feature set. This may be needed for other OpenCL application domains, and it can be easily integrated into our model. Finally, instead of using raw features, we group several features to form combined normalized features that carry more information than their parts (Table IIb).

Collecting Training Data. We use two sets of benchmarks to train our model. First, we use a collection of 47 OpenCL kernels taken from various sources: SHOC [Danalis et al. 2010], Parboil [UIUC 2013], NVIDIA CUDA SDK [NVIDIA Corp. 2013], and AMD Accelerated Parallel Processing SDK [AMD 2013]. These benchmarks are mostly single precision with only one kernel in each program, whereas the NAS benchmarks are double precision and have multiple kernels. We thus also add the NAS benchmarks to our training set but exclude the one for which we make a prediction (see Section 6.2).

Predictive Modeling. Our decision tree-based model is constructed using the C4.5 algorithm [Quinlan 1993]. The model is automatically built from training data by correlating features to the best-performing device (Figure 4). The model performs only on the generated OpenCL code. As such, it is independent of the type of the input program of our compiler. An example of a decision tree is given later in Figure 13, where a decision is made by comparing one of the combined features (Table IIb) to a threshold. If the feature is smaller than the threshold, the left subtree is traversed; otherwise, the right one is traversed. This is repeated until a leaf node is reached, labeled with one of the classes- "CPU" or "GPU"-tellingus which device to use.

\subsection{Runtime Deployment}

Once we have built the ML model as described previously, we can insert the model together with the syntax code features (extracted at compile time) to the generated code for any unseen, new programs so that the model can be used at runtime.

Updating Feature Values. At compile time, the OpenCL kernel is analyzed and code features are extracted and inserted to the generated program together with the trained ML model. As some loop bounds depend on the input, the compiler might be unable to determine certain feature values. These features are represented as static symbolic precomputation of loop bound variables, which will be updated using runtime values at runtime. If the loop bounds still cannot be determined at the time the prediction function is called, we simply use the average loop bound value as an estimation.

Version Selection. Figure 5 depicts the process of version selection during runtime. The first time a kernel is called, the built-in predictive model selects a code version for execution (line 12 in Figure 3). It uses instantiated feature values to predict the best computing device to use and sets function pointers to the corresponding code version. In our current implementation, prediction happens once during a program's execution. The overhead of prediction is negligible (a few microseconds). This cost is included in our later results. 


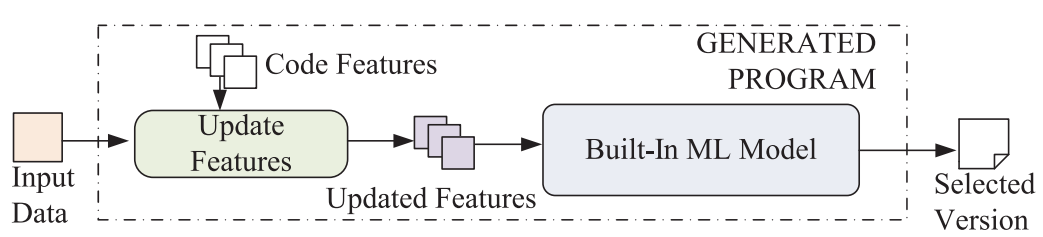

Fig. 5. Runtime: selecting a code version at execution time. The generated program first updates features based on input data and passes the updated features to the ML model. The model then predicts the best device (CPU or GPU) to run the program and selects a code version (OpenMP or OpenCL) for execution.

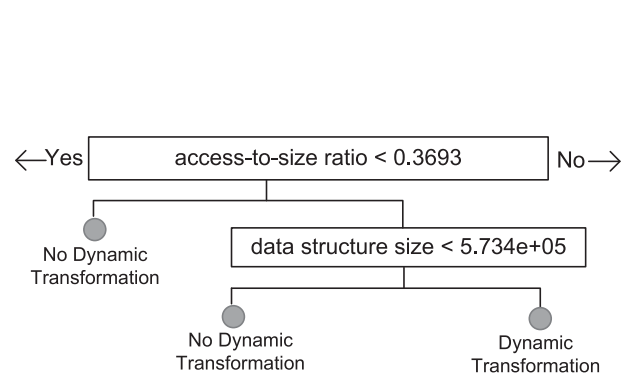

(a) NVIDIA GeForce GTX580

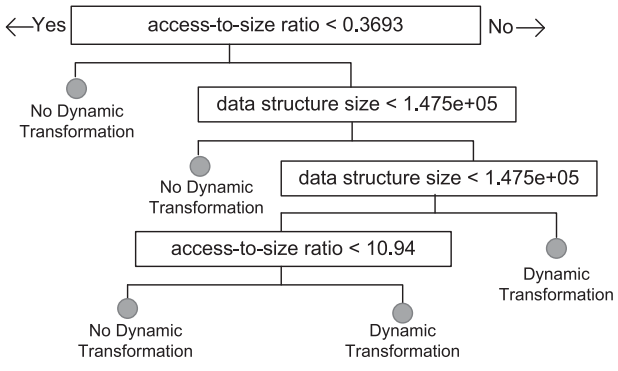

(b) AMD Radeon HD7970

Fig. 6. The automatically constructed decision trees for dynamic index reordering transformation on NVIDIA GeForce and AMD Radeon GPU platforms.

\subsection{A Model for Dynamic Index Reordering}

In Section 4.1, we described the dynamic index reordering transformation. Although this transformation can greatly improve performance on the GPU, it can also lead to slowdowns if the cost of reordering the data is higher than the benefits. Because the point at which the benefits outweigh the costs is highly machine dependent, we are using a portable machine learning approach that can be easily adapted to different systems. Similar to predicting the mapping, we use a decision tree classifier. The features are the size of the data structure and the ratio between the number of accesses to the data structure and its size.

We developed a set of microbenchmarks and use them to obtain the training data for this problem. ${ }^{2}$ We measure the execution time with and without applying dynamic index reordering to determine whether it is beneficial in each case. Evaluating the benchmarks and then building the decision tree model takes less than half an hour.

The resulting model is embedded into each output program because array dimensions and loop bounds may not be known at compile time. We thus keep two versions of each candidate kernel: the original one and one with accesses reordered. At runtime, one of them gets picked by the model.

Model. Figure 6 shows the decision trees for dynamic index reordering transformation on the two GPU platforms. We used the C4.5 algorithm [Quinlan 1993] to construct the decision tree model. The model is automatically built from training data that consists of data points describing a particular scenario for dynamic index reordering and whether it is beneficial or not. Each scenario is represented by two features: the size of the data structure and the ratio between the number of accesses and the size (corresponding to benefits over costs).

\footnotetext{
${ }^{2}$ We opted for microbenchmarks because the amount of training data from real applications is limited.
} 
Table III. The Primary Evaluation Hardware Platforms

\begin{tabular}{llll}
\hline & \multicolumn{1}{c}{ Intel CPU } & \multicolumn{1}{c}{ NVIDIA GPU } & \multicolumn{1}{c}{ AMD GPU } \\
\hline Model & Core i7 3820 & GeForce GTX 580 & Radeon 7970 \\
Core Clock & $3.6 \mathrm{GHz}$ & $1,544 \mathrm{MHz}$ & $925 \mathrm{MHz}$ \\
Core Count & $4(8 \mathrm{w} / \mathrm{HT})$ & 512 & 2,048 \\
Memory & $12 \mathrm{~GB}$ & $1.5 \mathrm{~GB}$ & $3 \mathrm{~GB}$ \\
Peak Performance & $122 \mathrm{GFLOPS}$ & $1,581 \mathrm{GFLOPS}$ & $3,789 \mathrm{GFLOPS}$ \\
\hline
\end{tabular}

\section{EXPERIMENTAL METHODOLOGY}

\subsection{Platforms and Benchmarks}

Our main experiments were performed on two CPU-GPU systems: both use an Intel Core i7 six-core CPU. One system contains an NVIDIA GeForce GTX 580 GPU, and the second contains an AMD Radeon 7970. Both run with the Ubuntu 10.10 64-bit OS. Table III gives detailed information on our platforms. We also evaluated our approaches on other GPU platforms, which are described in the sections where the results are presented.

All eight of the NAS parallel benchmarks (v2.3) were used for evaluation. We used the OpenMP C translation of the NAS 2.3 benchmark suite derived from the Omni compiler project [Omini Compiler Project 2009]. Unlike many GPU benchmarks that are single precision, all benchmarks except is are double precision programs.

\subsection{Methodology}

We considered all input sizes (S, W, A, B, C) for each NAS benchmark as long as the required memory fit into the GPU memory. All programs have been compiled using GCC 4.4.1 with the "-O3" option. Each experiment was repeated five times, and the average execution time was recorded. The variation of runtime is small, less than $5 \%$.

We use leave-one-out cross validation to train and evaluate our ML model for predicting the best computing device. This means that we remove the target program to be predicted from the training program set and then build a model based on the remaining programs. We repeat this procedure for each NAS benchmark in turn. It is a standard evaluation methodology, providing an estimate of the generalization ability of an ML model in predicting an unseen program. This approach is not necessary for the dynamic index reordering model because we use microbenchmarks as training data rather than the programs themselves.

Since OpenCL programs can also run on the CPU, an interesting question is to consider running the OpenCL programs on the CPU. However, for the benchmarks we used, doing so (with CPU-specific optimization) does not give advantages over other schemes considered in this article. On only one occasion, bt.W, such a scheme gives slightly better performance ( $8 \%$ faster) than other schemes. We also observed this on the CPU-tuned SNU OpenCL implementation. This may change when using other sets of benchmarks, hardware platforms, or OpenCL runtime and is an interesting future research direction.

\section{EXPERIMENTAL RESULTS}

In this section, we evaluate our approach on several heterogeneous systems for the NAS parallel benchmark suite. We first show the performance of our predictive modeling approach compared to always using the multicore CPU or the GPU. This is followed by a comparison to two state-of-the-art GPU code generation approaches: OpenMPC [Lee et al. 2009] and OpenACC [Portland Group 2010]. We then compare our approach against a manual OpenCL implementation of the NAS benchmark suite [Seo et al. 2011]. Next, we provide detailed analysis of our approach, including the performance 


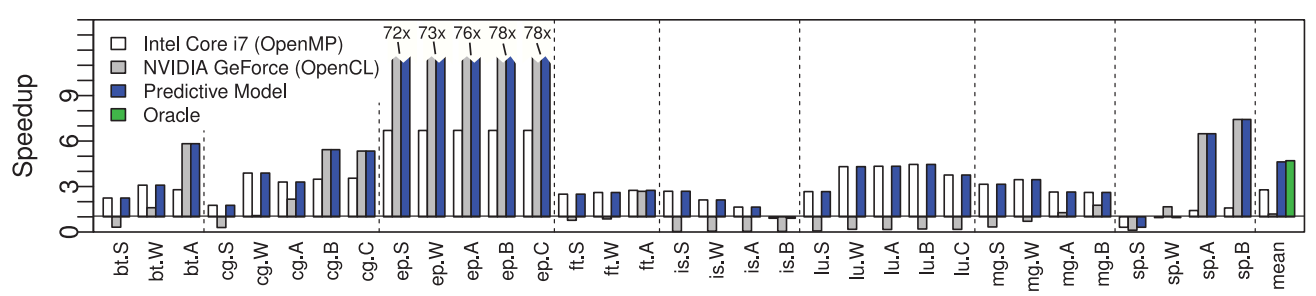

Fig. 7. Performance of OpenMP on an Intel CPU, OpenCL on an NVIDIA GeForce GTX580 GPU, and the version selected by our predictive model. The predictive model outperforms the CPU-only approach by $1.69 \times$ and the GPU-only approach by $3.9 \times$.

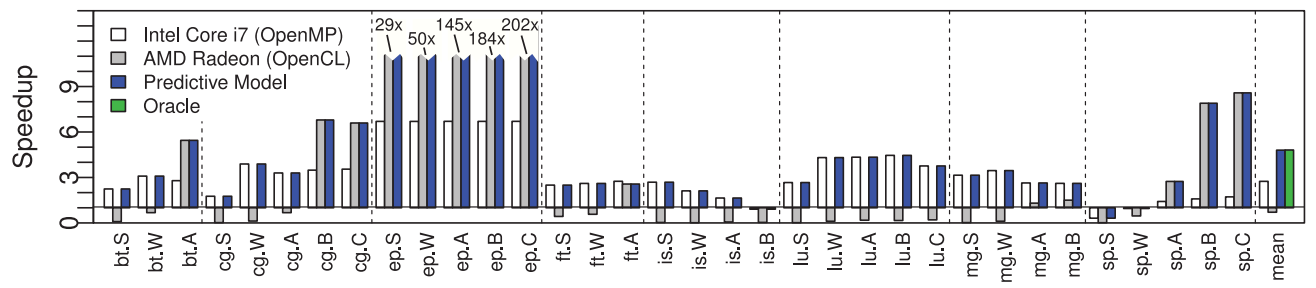

Fig. 8. Performance of OpenMP on an Intel CPU, OpenCL on an AMD Radeon HD7970 GPU, and the version selected by our predictive model. The predictive model outperforms the CPU-only approach by $1.76 \times$ and the GPU-only approach by $6.8 \times$.

breakdown of different optimization strategies, OpenCL runtime, and a close look of our predictive models. Finally, we present a brief evaluation of our approach on two heterogeneous systems with integrated GPUs.

\subsection{Overall Performance}

Figures 7 and 8 show speedups for the NAS benchmarks on the two heterogeneous systems described in Section 6. For each benchmark-input pair, the multicore CPU performance, the GPU performance, and the performance of the device selected by our predictor is shown. The last column represents the average performance (using the geometric mean) of each approach as well as that of the "oracle," which always picks the best device in each case. The performance numbers presented are speedups over single-core execution.

On both systems, significant speedups can be achieved by selecting the right device: CPU or GPU. When always selecting the faster of the two, speedups of $4.70 \times$ on the NVIDIA system and $4.81 \times$ on the AMD system can be achieved. This compares to $2.78 \times$ and $2.74 \times$ when always using the $\mathrm{CPU}^{3}$ and $1.19 \times$ and $0.71 \times$ on the GPU.

The results show that speedups vary dramatically between the CPU and GPU, and none of the devices consistently outperforms the other. On ep, for example, an embarrasingly parallel benchmark, the GPU clearly outperforms the multicore CPU: up to $11.6 \times$ on NVIDIA and $30.2 \times$ on AMD. However, on other benchmarks, such as is or $1 \mathrm{u}$, the CPU is significantly faster. In the case of $1 \mathrm{u}$, this is because the OpenMP version exploits pipeline parallelism using a combination of asynchronous parallel loops and a bit array to coordinate pipeline stages. The current SIMD-like execution models of GPUs are not designed to exploit this type of parallelism. The is benchmark does not perform a significant amount of computation, and GPU execution is dominated by

\footnotetext{
${ }^{3}$ Even though the same CPU was used in both cases, the numbers vary slightly because the benchmark sets are different due to memory constraints on the GPUs.
} 
communication with the host memory. This leads to underutilization of the GPU and thus bad performance.

For benchmarks bt, cg, and sp, we observe that the CPU is faster for small inputs but the GPU is better on larger input sizes. This behavior is to be expected because GPUs require large amounts of computation to fully exploit their resources. On small inputs, the overheads of communication with the host dominate the overall runtime when using the GPU. A similar pattern is shown for $\mathrm{ft}$ and $\mathrm{mg}$ : GPU performance is stronger for larger inputs. However, the GPU is not able to beat the CPU even for the largest input sets. For is, because the program does not have enough parallelism, it is actually not worthwhile to run it in parallel for any given dataset on our platforms. This is also reported in other studies [Tournavitis et al. 2009].

These observations show the need for a careful mapping of applications to devices. Our model for predicting the mapping is able to choose the correct device almost all of the time. On the NVIDIA system, it incorrectly picks the GPU for benchmark sp.W, and on the AMD system, it picks the GPU for ft.A even though the CPU is faster. Overall, we are able to achieve speedups of $4.63 \times$ and $4.80 \times$, respectively. This is significantly better than always choosing the same device and not far off the performance of the "oracle."

As can be seen, the best performance depends on the platform, transformations available, and data sizes. Our scheme is able to predict the right option, achieving $95 \%$ of oracle performance on the NVIDIA GTX580 system and even $99 \%$ on the AMD HD7970 system.

Prediction Accuracy. Our predictive model picks the correct device in 32 of the 33 cases (except for sp.W) on the NVIDIA system (97\% accuracy) and in 33 of the 34 cases (except for ft.A) on the AMD system (97\% accuracy).

\subsection{Comparison to State-of-the-Art GPU Code Generators}

We compared our approach to two automatic GPU code generation systems: (1) OpenMPC [Lee et al. 2009], which translates OpenMP to CUDA, and (2) the PGI OpenACC compiler (v 14.4) with the "-fast" and accelerator-specific optimization flags [OpenACC 2013; Wolfe 2010; Portland Group 2010]. Because the PGI compiler fails to directly compile the OpenMP version of the NAS benchmark suite, we used a GPU-specific OpenACC implementation of the same benchmark suite developed by independent developers [PathScale Inc. 2013]. Because OpenMPC generates CUDA instead of OpenCL code, we only evaluated it on the NVIDIA platform. Note that we were unable to generate code for benchmarks is, lu, and mg using OpenMPC. The results are shown in Figure 9.

With the exception of ep, the OpenMPC-generated code performs poorly compared to our approach. It only achieves a mean speedup of $0.42 \times$ - that is, slower than sequential execution. The main reason is that OpenMPC does not perform data transformation, leading to uncoalesced memory accesses in many cases. On average, our approach outperforms OpenMPC by a factor of 10 .

The PGI OpenACC compiler also gives overall slowdown performance. Note that by excluding is, lu, and mg, which OpenMPC fails to compile, the PGI OpenACC compiler actually gives better overall performance than OpenMPC ( 0.7 vs. 0.4$)$. OpenACC outperforms OpenMPC on the cg benchmark but delivers poorer performance than our approach on both platform. On average, it gives slowdown performance instead of a $4.18 \times$ and $3.9 \times$ speedup achieved by our approach on the NVIDIA and AMD platform, respectively. Because the PGI OpenACC compiler is an closed source software, we cannot get deep insights of its implementation. Instead, we used the AMD CodeXL profiling tool [AMD 2014] to analyze the generated OpenACC code on the 


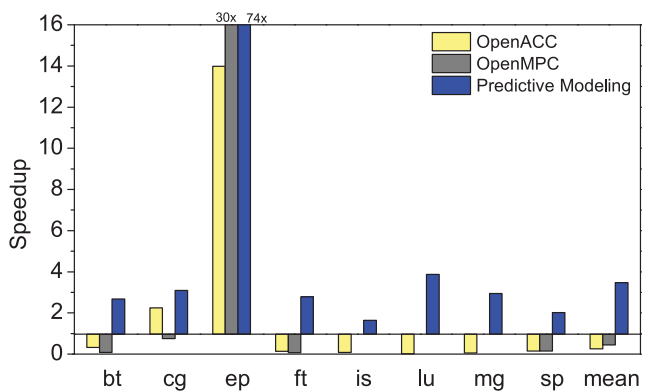

(a) NVIDIA GeForce GTX580

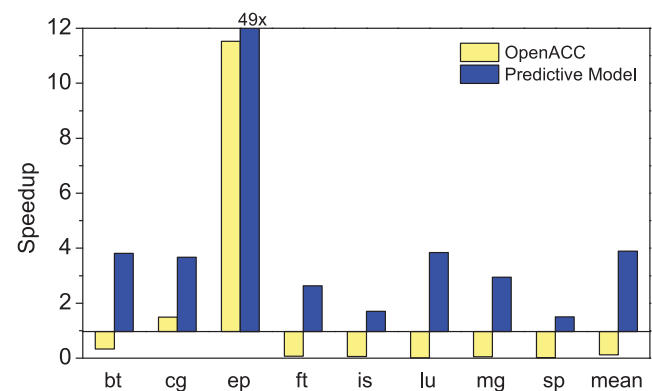

(b) AMD Radeon HD7970

Fig. 9. Speedup averaged across inputs of the OpenMPC compiler (NVIDIA only), the manual SNU implementation of the NAS benchmark suite and our predictive modeling.

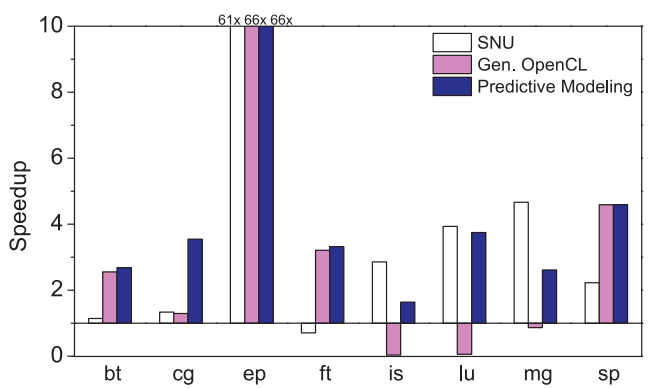

(a) NVIDIA GTX480

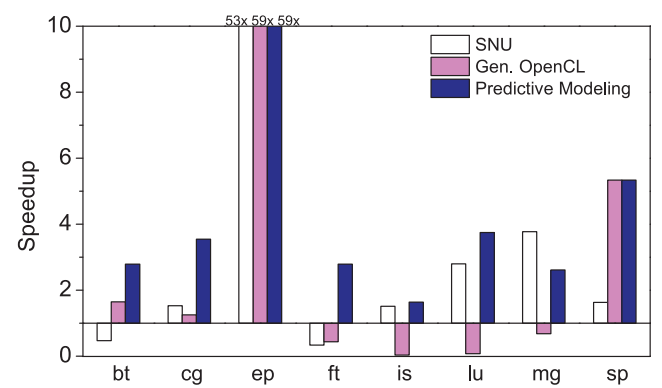

(b) AMD HD6970

Fig. 10. Speedup of the SNU implementation, our generated OpenCL code, and the predictive modelingbased approach for the largest possible input size. This experiment was performed on two GPUs that the SNU developers have used for testing and evaluation.

AMD GPU. We discovered that the OpenACC version has significantly longer OpenCL kernel execution time compared to our approach. This may attribute to the lacking of array index transformations to reduce the noncoalesced memory accesses of OpenCL kernels. For some benchmarks, such as $\mathrm{lu}$ and is, the GPU gives no advantage and the OpenACC runtime does not dynamically choose computing devices, thus leading to poor performance. On average, our approach is $13 \times$ and $32 \times$ faster than the OpenACC implementation on the NVIDIA and AMD platforms, respectively.

\subsection{Comparison to Hand-Coded Implementation}

Figure 10 compares the generated OpenCL code to the handwritten SNU implementation [Seo et al. 2011]. This provides independently handwritten OpenCL implementations of the NAS parallel benchmarks. We selected the largest possible input size for each benchmark. To provide a fair comparison, the experiments were carried out on two platforms where the developers have tested the code: a NVIDIA GTX 480 GPU and an AMD Radeon HD6970 GPU.

The data show mixed results. For benchmarks bt, sp, and ft, our automatically generated code outperforms the handwritten code. This is mainly due to the data restructuring performed by our compiler, including dynamic index reordering, which is especially important for benchmarks bt and sp. For cg and ep, the speedups for both OpenCL code versions are similar, but our predictive model outperforms SNU NPB on $\mathrm{cg}$ by selecting the right computing device (CPU) and using the OpenMP version. 
On the remaining benchmarks-is, $1 \mathrm{u}$, and $\mathrm{mg}$ - our generated code is not as good as the SNU implementation. The SNU version of lu uses a different algorithm than the original OpenMP code [Seo et al. 2011]. Their implementation uses a hyperplane algorithm that is much more suited to GPU execution. Changing the algorithm is out of the scope of our approach. For is, the SNU implementation uses atomic operations to compute a histogram and a parallel prefix sum algorithm that is not exposed in the OpenMP code and is not supported by our current implementation. The code for mg works on highly irregular multidimensional data structures. In the generated code, these data structures are flattened and indirection in each dimension is used to navigate through the data. The SNU implementation uses a different approach that requires a single level of indirection that leads to vastly improved performance. Nonetheless, our ML model is able to pick the right computing device for those benchmarks, and the performance gap between the manual implementation and our predictive modeling-based approach is not significant.

Overall, our generated OpenCL code performs well. The hand-coded versions generally only perform better when algorithmic changes or code restructuring is performed, which is difficult to achieve by an automatic compiler without human involvement.

\subsection{Performance Breakdown}

Figure 11 shows the impact of the transformations described in Section 4 whenever they were applicable to a benchmark.

$B T$. As shown in Section 2, bt contains many multidimensional arrays and deeply nested loops. Without applying any of the transformations, performance is thus poor on the GeForce system. But even when applying loop interchange and static data transformations, the performance only improves marginally (up to $0.81 \times$ ). This is because there are several large sections of the program that require different data layouts to achieve memory coalescing and perform well on the GPU. Hence, when also applying dynamic array index reordering, performance improves markedly. However, when compared to CPU performance, we only see speedups for large input sizes W and A. On the Radeon system (Figure 11(e)), a similar behavior can be observed.

$C G$. This benchmark only contains one-dimensional arrays and is thus not amenable to loop interchange or data transformations. However, as shown in Figure 11(b) and (f), good performance on GPUs can already be achieved without any transformations. For small input sizes, slowdowns can be observed due to insufficient amounts of computation. But for large input sizes, the GPU outperforms the CPU with speedups of up to $5.56 \times$ and $6.79 \times$ on the two GPU architectures.

$E P$. Figure 11(c) and (g) show results for ep, an embarrassingly parallel benchmark that performs a significant amount of computation per work item. Its data structures are one-dimensional arrays, and data transformations are thus not applicable. However, even without any transformations, speedups of up to $78 \times$ on the GeForce and $202 \times$ on the Radeon systems over single-core execution are observed. This compares to speedups of only up to $10 \times$ on the Core $i 7$.

$F T$. For the ft benchmark, we see speedups on the GPU of up to $2.6 \times$ for large input sizes (see Figure 11(d) and (h)). With small inputs using the GPU leads to slowdowns, even after applying data transformations.

IS. On both architectures, significant slowdowns are shown for GPU execution on all input sizes-from $0.03 \times$ to $0.78 \times$ on the GeForce GPU and from 0.01 to 0.04 on the Radeon GPU (Figure 11(i) and (m)). But even on the CPU, only small speedups can be achieved. This is dominated by communication with the host memory. Furthermore, 


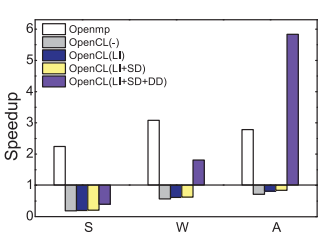

(a) bt on GeForce GTX580

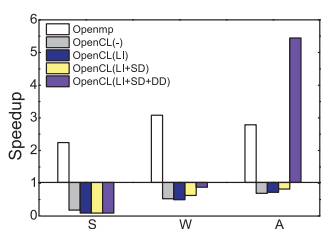

(e) bt on Radeon HD7970

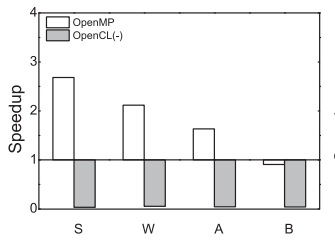

(i) is on GeForce GTX580

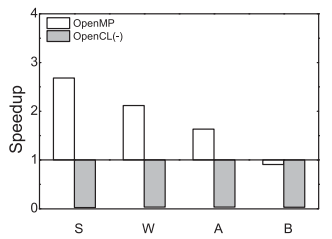

(m) is on Radeon HD7970

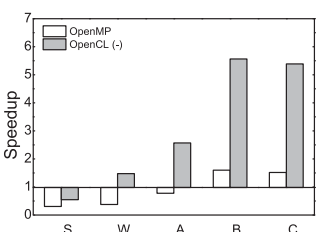

(b) cg on GeForce GTX580

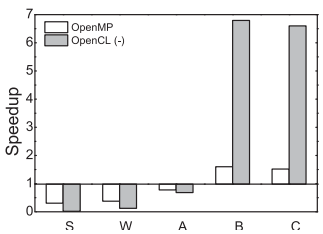

(f) cg on Radeon HD7970

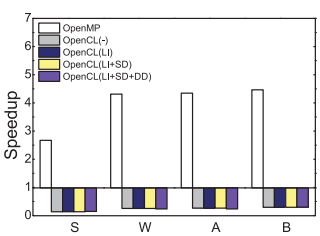

(j) lu on GeForce GTX580

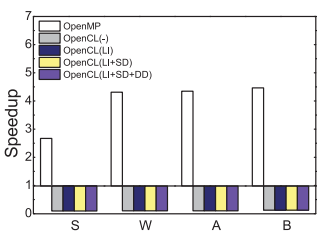

(n) Iu on Radeon HD7970

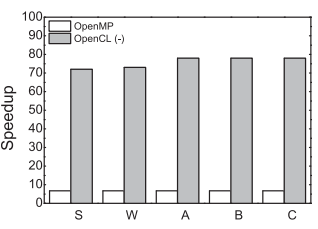

(c) ep on GeForce GTX580

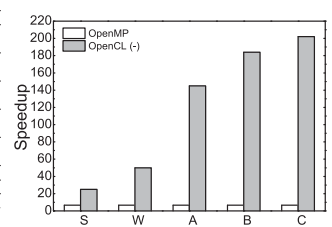

(g) ep on Radeon HD7970

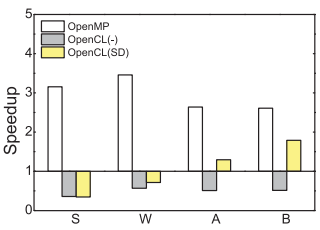

(k) mg on GeForce GTX580

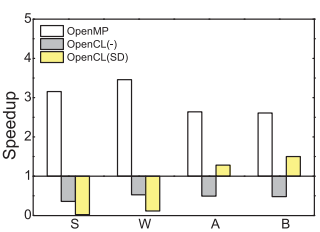

(o) mg on Radeon HD7970

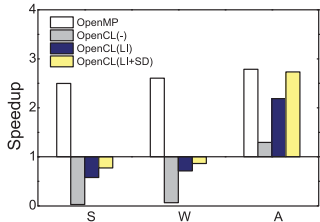

(d) ft on GeForce GTX580

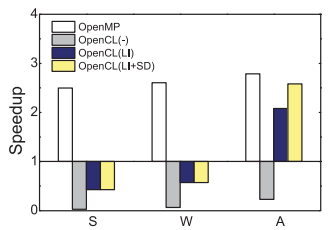

(h) $\mathrm{ft}$ on Radeon HD7970

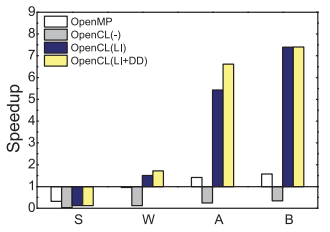

(l) sp on GeForce GTX580

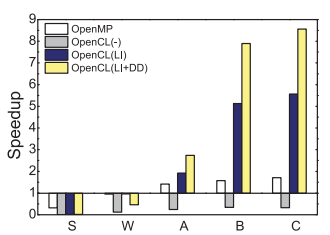

(p) sp on Radeon HD7970

Fig. 11. Speedup over single-core execution on Core i7 (OpenMP) and both the NVIDIA GTX580 and AMD HD7970 systems (OpenCL). Where applicable, we show the performance of the transformation steps. LI, loop interchange; SD, static data transformations; DD, dynamic data transformations.

each OpenCL work item needs to keep a private copy of a large array, which means that the total number of work items is limited by the GPU memory. This leads to underutilization of the GPU, which further reduces performance. Data transformations are not applicable to this benchmark because it only works on one-dimensional arrays.

$L U$. Even though the OpenMP code shows good speedups on the CPUs, performance on GPUs is poor despite applying data transformations (Figure $11(\mathrm{j})$ and $(\mathrm{n})$ ). $\mathrm{lu}$ is a complex program, and its OpenMP version exploits pipeline parallelism using a combination of asynchronous parallel loops and a bit array to coordinate pipeline stages. The current SIMD-like execution models of GPUs are not designed to exploit this type of parallelism. As a result, large parts of lu cannot utilize the GPU's massive parallel processing units and have to be serialized, which explains the slowdown on the GPUs (as can be seen from the expensive host-device communication shown in Figure 12).

$M G$. For this benchmark, we see that speedups are only achieved for large input sizes and after data transformations have been applied (Figure 11(k) and (o)). However, on none of the architectures does the GPU outperform the CPU. 


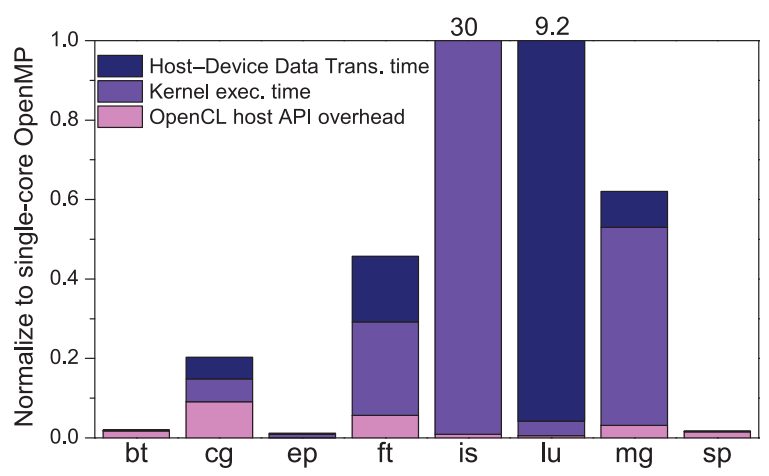

Fig. 12. OpenCL time breakdown on the AMD HD7970. Each category is normalized to the sequential execution of the program (lower is better). This diagram includes the host-device data transfer time, OpenCL kernel time, and OpenCL host API overhead.

$S P$. Similar to bt, dynamic data realignment is required to unlock the best performance for benchmark sp (Figure 11(l) and (p)). Static data transformations cannot improve on the original data layout. Speedups of up to $8.6 \times$ on the GeForce GPU and up to $4.1 \times$ on the Radeon GPU are shown for large input sizes. This compares to speedups of up to $2.0 \times$ on the CPU. For small data sizes, however, the CPU outperforms the GPU.

\subsection{Breakdown of OpenCL Runtime}

Figure 12 shows the breakdown of OpenCL execution time for each benchmark (with the largest possible input size) on the AMD HD7970 system. This information was collected using the AMD CodeXL profiling tool [AMD 2014]. The time is normalized to single-core execution of the original OpenMP program (lower is better). Each stacked bar consists of host-device data transfer time, cumulative OpenCL kernel execution time, and overhead for executing OpenCL host APIs the CPU.

Some benchmarks, such as ep, bt, cg, and sp, can make effective use of the GPU with small kernel execution time and overall faster performance. Some benchmarks, by contrast, are not suitable for GPU execution. For example, for is, the small number of work items leads to underutilization of the GPU, and because each GPU processing unit is simpler and weaker than a CPU core, this results in longer kernel execution time. For $\mathrm{lu}$, the frequent CPU serial execution introduces intensive host-device communication and leads to slowdown performance. This figure shows that not all OpenCL programs can utilize the GPU and the available parallelism and the communication cost of task offloading are important factors when determining which device to use to run the program.

\subsection{Analysis of Predictive Models}

Figures 13 and 14 show the decision trees constructed for the two systems by excluding bt from the training set. The learning algorithm automatically places the most relevant features at the root level and determines the architecture-dependent threshold for each node. All of this is done automatically without the need of expert intervention.

As an example, the features for benchmark bt are shown in Table IV. ${ }^{4}$ We show the features both before and after applying data transformations according to the example shown in Section 2. This demonstrates the impact of the transformations on the mapping decision.

\footnotetext{
${ }^{4}$ The feature values for all benchmarks can be found at http://www.lancaster.ac.uk/staff/wangz3/data/taco_ omp2ocl_fatures.gz.
} 


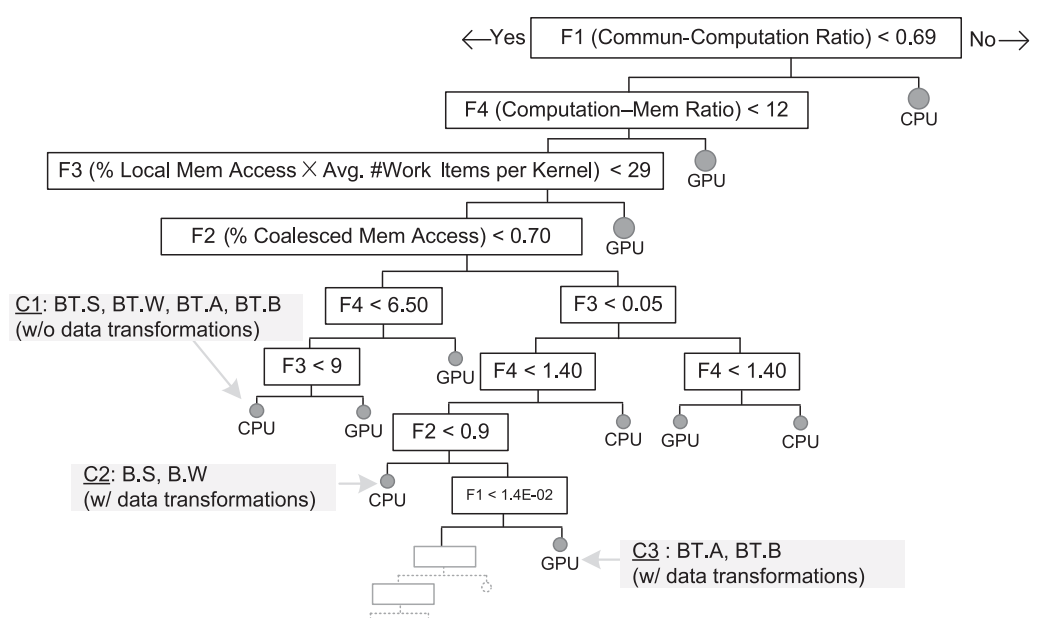

Fig. 13. The model used for bt on NVIDIA GeForce GTX 580. Predictions for bt with and without data transformations are marked as $\mathrm{C} 1, \mathrm{C} 2$, and C3.

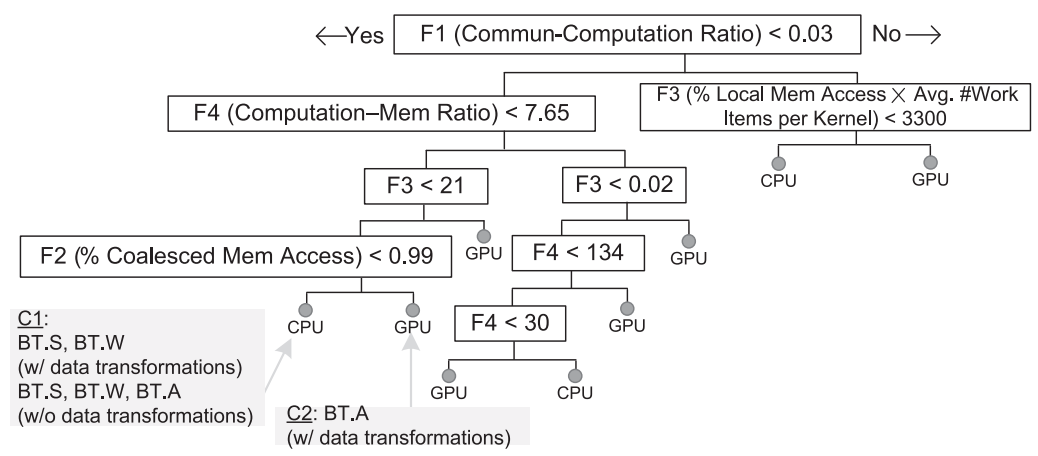

Fig. 14. The model used for bt on AMD Radeon HD7970. Predictions for bt with and without data transformations are marked as $\mathrm{C} 1$ and $\mathrm{C} 2$.

Table IV. Features of bt

(a) Without data transformations

\begin{tabular}{c|c|c|c|c}
\hline & F1 & F2 & F3 & F4 \\
\hline S & 0.0020 & 0 & 0 & 0.81 \\
W & 0.0004 & 0 & 0 & 0.82 \\
A & 0.0003 & 0 & 0 & 0.82 \\
\hline
\end{tabular}

(b) With data transformations

\begin{tabular}{c|c|c|c|c}
\hline & F1 & F2 & F3 & F4 \\
\hline S & 0.0020 & $\mathbf{0 . 7 3 1}$ & 0 & 0.81 \\
W & 0.0004 & $\mathbf{0 . 7 8 0}$ & 0 & 0.82 \\
A & 0.0003 & $\mathbf{0 . 9 9 9}$ & 0 & 0.82 \\
\hline
\end{tabular}

At the root of the tree in Figure 13, we look at the value for the communicationcomputation ratio (F1). In all versions, the value is far below the threshold. We thus proceed to the left subtree until reaching the fourth level of the tree. This node looks at the percentage of coalesced memory accesses (F2). Without data transformations, none of the accesses are coalesced and the left branch is taken, eventually leading to CPU execution. With data transformations, memory coalescing has been improved (see bold values in Table IVb). For input sizes $\mathrm{S}$ and W, the percentage of coalesced accesses is less than $80 \%$. For A, almost all accesses are coalesced due to dynamic index reordering (see Section 4.1). All values are above the threshold, so the right branch is taken. We follow the same branches until another node of F2 is reached. This time the threshold 


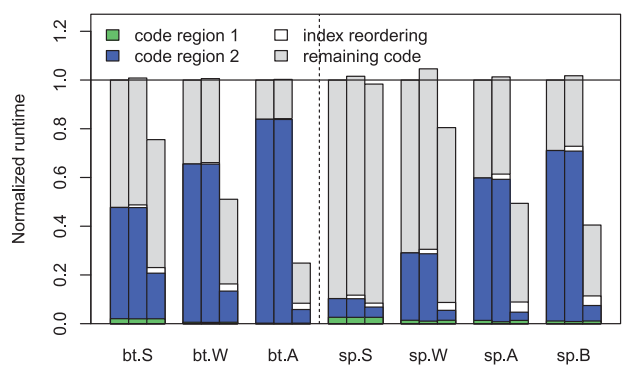

(a) NVIDIA GeForce GTX580

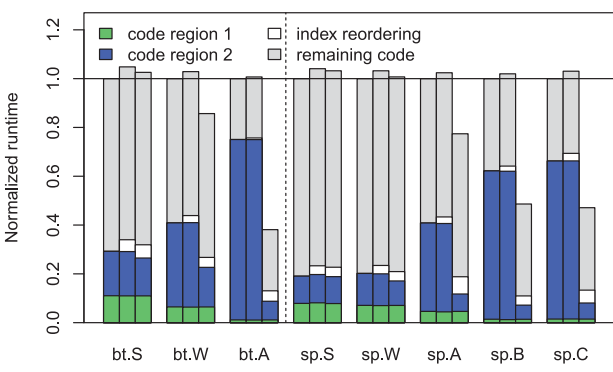

(b) AMD Radeon HD7970

Fig. 15. Performance impact of dynamic index reordering when applying the transformation to none, the first, or the second of the candidate regions. The runtime is broken up into the runtime for the two candidate code regions, the runtime of the transformation (if applicable), and the rest of the program.

is higher, namely 0.9 . For input sizes $\mathrm{S}$ and $\mathrm{W}$, the left branch is taken, which leads to execution on the CPU. For the larger input size A, we take the right branch and eventually reach a node predicting to run on the GPU. All programs get mapped to the right device.

Figure 14 shows the decision tree constructed for the AMD Radeon system. The first node also looks at the ratio between communication and computation. The threshold is lower (0.03); however, for all versions of bt, the ratio is still below the threshold. The same path is followed by all versions until the fourth level of the tree is reached. At this point, we look at the percentage of coalesced accesses. The versions without data transformations are mapped to the CPU because none of the accesses are coalesced. Even when applying data transformations, for input sizes $\mathrm{S}$ and $\mathrm{W}$ the value is below the threshold and the code gets mapped to the CPU. Only input size A is mapped to the GPU. All programs are again mapped to the right device.

\subsection{Dynamic Index Reordering}

The benchmarks bt and sp contain candidate regions for dynamic index reordering. Figure 15 shows the performance of the benchmarks with different input sizes when applying dynamic index reordering to none, the first, or the second of those regions. The performance is normalized to the runtime when the transformation is not applied. In each case, the runtime is broken up into the runtimes for the two candidate code regions, the overhead of the transformation (if applicable), and the rest of the program.

The first candidate region makes up only a small fraction of the overall runtime of both benchmarks; $1 \%$ to $3 \%$ on the NVIDIA and $1 \%$ to $11 \%$ on the AMD system. When applying dynamic index reordering here, the performance of this region barely improves because there are not many memory accesses that benefit from the transformation. The cost of reordering the data thus often outweighs the benefits, which leads to minor slowdowns overall.

The second region, on the other hand, makes up a larger chunk of the overall runtime. Applying dynamic index reordering significantly reduces the runtime of this region. Since the overhead of data reordering is comparatively small, big overall runtime reductions are achieved by applying the transformation to this region: up to $75 \%$ on the NVIDIA system and $62 \%$ on the AMD system.

Similar to predicting on which device to run a program, we also use decision trees to determine when dynamic index reordering is beneficial (see Section 5.4 for details). Applying this model for bt and sp achieves an accuracy of $79 \%$ on the NVIDIA and $94 \%$ on the AMD system. 


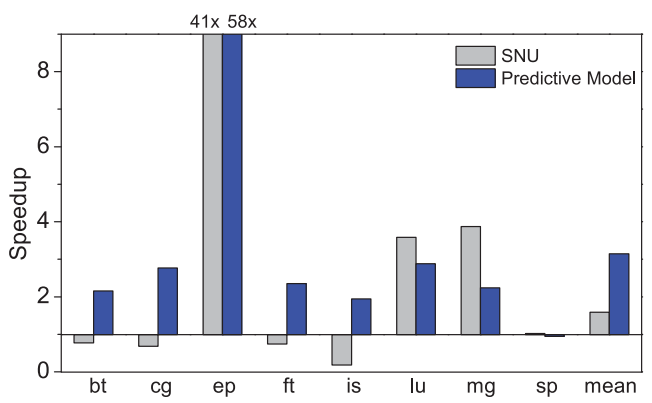

(a) AMD Llano

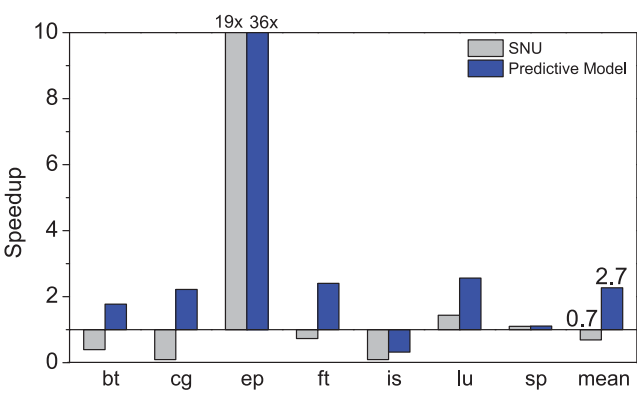

(b) Intel Ivy Bridge

Fig. 16. Speedup averaged across inputs of the manual SNU code and our model on systems with integrated GPUs.

\subsection{Performance on Integrated Systems}

GPU technology is constantly evolving. To check that our approach also works on new devices, we evaluated it on two systems with integrated GPUs: AMD Llano (A8-3850), and Intel Ivy Bridge (Core i5 3570K). Figure 16 shows a summary of the results using the SNU implementation and our predictive modeling approach. The manual SNU code only achieves speedups of $1.6 \times$ and $0.7 \times$ on average compared to $3.1 \times$ and $2.7 \times$ of our approach.

The integrated GPUs on these systems are less powerful than the discrete GPUs that we evaluated previously. This demonstrates even more the need for a model that only maps code to the GPU when it is beneficial. Integrated GPUs share the system memory with the CPU, making data movements between the devices cheaper or even unnecessary in the case of Intel IvyBridge. Because most benchmarks in the NAS parallel benchmark suite are compute intensive, this advantage does not lead to improved performance overall. Nonetheless, our portable ML-based approach is still able to achieve a speedup on average.

\section{RELATED WORK}

GPU Programming Languages. Programming support for GPUs has been a critical issue, and CUDA has been a significant reason for the success of general-purpose computing on GPUs. Impressive performance has been achieved with orders of magnitude improvements found for certain applications [Ryoo et al. 2008]. Despite the popularity of CUDA, it mainly targets NVIDIA GPUs and is not directly portable to other GPUs or more general heterogeneous architectures. OpenCL has the promise of having more general applicability at the cost of a potentially more complex programming model. Due to the relative immaturity of language implementations, recent work has focused on how to improve performance either using different code transformations [Lee and Eigenmann 2010] or partitioning across multiple GPUs [Kim et al. 2011]. The benchmarks considered are largely those well fitted to GPU architectures. In Bordawekar et al. [2010], Grewe and O'Boyle [2011], and Lee et al. [2010a], it was shown that although GPUs can often give significant performance for kernels, in some cases it is better not to use the GPU but to use the multicore instead.

High-Level Programming Models. There have been several different approaches to generating GPU code from simpler higher-level languages. In C to CUDA [Baskaran et al. 2010], nested loops are represented in a polyhedral framework that when mapped to the GPU give excellent performance. However, the set of programs that can be handled this way is extremely small and cannot be applied to more general parallel programs with arbitrary data dependence and control-flow structure. In Sponge [Hormati 
et al. 2011], again good performance is achieved. The benchmarks used were rewritten from the Parboil benchmarks (a CUDA benchmark suite) in StreamIt format, so again the benchmarks are those that are well fitted to GPUs. Apart from Gregg et al. [2010], none of these approaches uses OpenCL, and most of them rely on the user to provide a separate kernel version for CPUs and GPUs. We circumvent this problem by automatically generating multiversions of the input program and building a portable machine learning model to automatically select a code version at runtime.

Automatic Generation of GPU Programs. The OpenMPC compiler [Lee et al. 2009] translates OpenMP to CUDA programs. Unlike our approach, OpenMPC neither performs dynamic data transformations nor uses predictive modeling to select a code version across different GPU architectures. The OpenACC programming interface [OpenACC 2013] defines a set of compiler directives for expressing loop- and regionbased parallelism. Using an OpenACC enabling compiler, the parallelism can be translated into OpenCL or CUDA implementations or be offloaded onto an accelerator. In Baskaran et al. [2010], CUDA programs are automatically generated from sequential, affine $\mathrm{C}$ programs using the polyhedral model. In all preceding approaches, the code always gets executed on the GPU. Prior work on automatic generation of parallel GPU code from sequential programs also includes Par4ALL [Amini et al. 2012], PPCG [Verdoolaege et al. 2013], and that of Wang et al. [2014a]. Unlike our approach, they do not consider the problem of selecting the most suitable device from the host CPU and the GPU to run the code.

Optimizing GPU Programs. CGCM [Jablin et al. 2011] is a CPU-GPU communication system to optimize CUDA applications between the host and the GPU. In the following work [Jablin et al. 2012], DyManD was proposed to overcome the limitation of CGCM by replacing static analysis with a dynamic runtime system. DyManD is able to optimize programs that cannot automatically handle CGCM. CUDA-lite [Ueng et al. 2008] relies on programmer annotations to exploit GPU performance by coalescing memory accessing. Sung et al. [2010] propose a data layout transformation for structure grid programs (e.g., stencil code). The input to their tools are arrays that are in a restricted form. Dymaxion [Che et al. 2011] allows programmers to manually apply index reordering for CUDA programs. In contrast to Dymaxion, which has a single data layout for the entire program, our compiler automatically applies dynamic index reordering to parts of the program when such a transformation is profitable. Furthermore, in Dymaxion, index reordering can only be applied when transferring data from the host to the GPU, whereas our technique is applied when the data is already on the GPU. Recently, Kayiran et al. [2013] proposed a dynamic scheduling approach to determine the optimal number of GPU threads to reduce the resource contention on the GPU. The StarPU runtime system provides a unified framework for scheduling numerical kernels on heterogeneous systems [Augonnet et al. 2011]. StarPU requires the developers to provide a cost model for each task and uses heuristics to dynamically schedule parallel tasks. This fine-grained, runtime-based approach complements our compiler-based approach.

Data Layout Transformation. Data layout is crucial to application performance, and there is an extensive body of work for data layout transformation on CPUs. A good review of existing CPU techniques could be found in the report of Karlsson [2009]. $\mathrm{DL}$ is a runtime data layout transformation framework for GPU applications [Sung et al. 2012]. It offers several useful data layout transformations, such as transforming array-of-structures to/from structure-of-arrays and in-place layout conversion. Prior work also includes Dymaxion [Che et al. 2011] and in-place matrix transposition [Sung et al. 2014; Gustavson et al. 2012]. Impressive results have been achieved using those 
approaches. Unlike prior work that relies on the programmer to determine the cost and benefit of data transformations, our approach uses machine learning to automatically learn a cost model that automatically decides whether a transformation is beneficial for a given program, input, and hardware.

Mapping Parallel Programs. Most prior research on parallelism mapping has focused on building platform-specific heuristics [Ramanujam and Sadayappan 1989; Huang et al. 2009] for a certain class of platforms. Such an approach is tightly coupled with a specific architecture and as a result cannot adapt to the fast-evolving GPU architecture. Our predictive model, on the other hand, can adapt to the change of hardware and compilers by automatically learning from data. Some other approaches use iterative compilation and search to tune GPU programs [Datta et al. 2008]. However, these approaches can lead to excessive profile runs for a single program. Our recent work [Grewe et al. 2013b; Wen et al. 2014] takes the machine learning-based approach further and automatically partitions GPU kernels between the CPU and GPU in the presence of workload contention (i.e., multiple programs compete for the shared computing resources). Our scheme achieves significant speedups over a GPUonly scheme, demonstrating the advantages of machine learning-based parallelism mappings.

Predictive Modeling. In addition to optimizing sequential programs [Cooper et al. 1999], recent studies have shown that predictive modeling is effective in optimizing parallel programs [Wang and O'Boyle 2009, 2010, 2013; Collins et al. 2013; Emani et al. 2013; Wang et al. 2014b] and scheduling parallel workload [Grewe et al. 2011]. The Qilin [Luk et al. 2009] compiler uses offline profiling to create a regression model that is employed to predict a data parallel program's execution time. Unlike Qilin, our approach does not require any profiling runs during compilation. Recently, machine learning has been used to predict the best mapping of a single OpenCL kernel [Grewe and O'Boyle 2011; Ogilvie et al. 2014]. In contrast to this work, our compiler automatically transforms large OpenMP programs into OpenCL-based programs and predicts whether the OpenMP or OpenCL code gives the best performance on the system.

\section{CONCLUSION AND FUTURE WORK}

This article has described a compilation approach that takes shared memory programs written in OpenMP and outputs OpenCL code targeted at GPU-based heterogeneous systems. The proposed approach uses loop and array transformations to improve the memory behavior of the generated code. OpenCL is a portable standard, and we evaluate its performance on different platforms: NVIDIA GeForce and AMD Radeon discrete GPUs, as well as integrated GPUs. This approach was applied to the whole NAS parallel benchmark suite, where we show that in certain cases the OpenCL code generated can produce significant speedups (up to $202 \times$ ). However, GPUs are not best suited for all programs, and in some cases it is more profitable to use the host multicore instead. We developed an approach based on machine learning that determines for each new program whether the multicore CPU or the GPU is the best target. If the multicore is selected, we run the appropriate OpenMP code, as it currently outperforms OpenCL on multicores. This model is learned on a per-platform basis, and we demonstrate that the model adapts to different platforms and achieves consistent prediction accuracy. We thus build on the portability of OpenCL as a language by developing a system that is performance portable as well.

Future work will examine a much greater range of program optimizations. In particular, we wish to examine exploitation of the GPU memory hierarchy and apply autovectorization; both of these are likely to benefit the AMD Radeon and other GPUs. 


\section{REFERENCES}

AMD. 2013. AMD/ATI Stream SDK. Retrieved October 17, 2014, from http://www.amd.com/stream/.

AMD. 2014. CodeXL-Powerful Debugging, Profiling \& Analysis. Retrieved October 17, 2014, from developer.amd.com/tools-and-sdks/opencl-zone/codexl/.

Mehdi Amini, Onig Goubier, Serge Guelton, Janice Onanian McMahon, François Xavier Pasquier, Grégoire Péan, and Pierre Villalon. 2012. Par4All: From convex array regions to heterogeneous computing. In Proceedings of the 2nd International Workshop on Polyhedral Compilation Techniques (IMPACT'12).

Cédric Augonnet, Samuel Thibault, Raymond Namyst, and Pierre-André Wacrenier. 2011. StarPU: A unified platform for task scheduling on heterogeneous multicore architectures. Concurrency and Computation: Practice and Experience 23, 2, 187-198.

Muthu M. Baskaran, J. "Ram" Ramanujam, and Ponuswamy Sadayappan. 2010. Automatic C-to-CUDA code generation for affine programs. In Compiler Construction. Lecture Notes in Computer Science, Vol. 6011. Springer, 244-263.

Rajesh Bordawekar, Uday Bondhugula, and Ravi Rao. 2010. Believe it or not! Multi-core CPUs can match GPU performance for a FLOP-intensive application! In Proceedings of the 19th International Conference on Parallel Architectures and Compilation Techniques (PACT'10). 537-538.

Gautam Chakrabarti, Vinod Grover, Bastiaan Aarts, Xiangyun Kong, Manjunath Kudlur, Yuan Lin, Jaydeep Marathe, Mike Murphy, and Jian-Zhong Wang. 2012. CUDA: Compiling and optimizing for a GPU platform. Procedia Computer Science 9, 1910-1919.

Shuai Che, Jeremy W. Sheaffer, and Kevin Skadron. 2011. Dymaxion: Optimizing memory access patterns for heterogeneous systems. In Proceedings of the International Conference for High Performance Computing, Networking, Storage, and Analysis (SC'11). Article No. 13.

Alexander Collins, Christian Fensch, Hugh Leather, and Murray Cole. 2013. MaSiF: Machine learning guided auto-tuning of parallel skeletons. In Proceedings of the 20th International Conference on High Performance Computing (HiPC'13). 186-195.

Keith D. Cooper, Philip J. Schielke, and Devika Subramanian. 1999. Optimizing for reduced code space using genetic algorithms. In Proceedings of the ACM SIGPLAN Workshop on Languages, Compilers, and Tools for Embedded Systems (LCTES'99). 1-9.

Anthony Danalis, Gabriel Marin, Collin McCurdy, Jeremy S. Meredith, Philip C. Roth, Kyle Spafford, Vinod Tipparaju, and Jeffrey S. Vetter. 2010. The scalable heterogeneous computing (SHOC) benchmark suite. In Proceedings of the 3rd Workshop on General-Purpose Computation on Graphics Processing Units (GPGPU'10). 63-74.

Kaushik Datta, Mark Murphy, Vasily Volkov, Samuel Williams, Jonathan Carter, Leonid Oliker, David Patterson, John Shalf, and Katherine Yelick. 2008. Stencil computation optimization and auto-tuning on state-of-the-art multicore architectures. In Proceedings of the ACM/IEEE Conference on Supercomputing (SC'08). Article No. 4.

Alexandre E. Eichenberger, Peng Wu, and Kevin O'Brien. 2004. Vectorization for SIMD architectures with alignment constraints. In Proceedings of the ACM SIGPLAN Conference on Programming Language Design and Implementation (PLDI'04). 82-93.

Murali K. Emani, Zheng Wang, and Michael F. P. O’Boyle. 2013. Smart, adaptive mapping of parallelism in the presence of external workload. In Proceedings of the IEEE/ACM International Symposium on Code Generation and Optimization (CGO'13). 1-10.

Chris Gregg, Jeff Brantley, and Kim Hazelwood. 2010. Contention-Aware Scheduling of Parallel Code for Heterogeneous Systems. Technical Report. Department of Computer Science, University of Virginia.

Dominik Grewe and Michael O’Boyle. 2011. A static task partitioning approach for heterogeneous systems using OpenCL. In Proceedings of the 20th International Conference on Compiler Construction: Part of the Joint European Conferences on Theory and Practice of Software (CC'11/ETAPS'11). 286305.

Dominik Grewe, Zheng Wang, and Michael F. P. O’Boyle. 2013a. Portable mapping of data parallel programs to OpenCL for heterogeneous systems. In Proceedings of the IEEE/ACM International Symposium on Code Generation and Optimization (CGO'13). 1-10.

Dominik Grewe, Zheng Wang, and Michael F. P. O’Boyle. 2013b. OpenCL task partitioning in the presence of GPU contention. In Languages and Compilers for Parallel Computing. Lecture Notes in Computer Science, Vol. 8664. Springer, 87-101.

Dominik Grewe, Zheng Wang, and Michael F. P. O’Boyle. 2011. A workload-aware mapping approach for data-parallel programs. In Proceedings of the 6th International Conference on High Performance and Embedded Architectures and Compilers (HiPEAC'11). 117-126. 
Fred Gustavson, Lars Karlsson, and Bo Kågström. 2012. Parallel and cache-efficient in-place matrix storage format conversion. ACM Transactions on Mathematical Software 38, 3, Article No. 17.

Amir Hormati, Mehrzad Samadi, Mark Woh, Trevor N. Mudge, and Scott A. Mahlke. 2011. Sponge: Portable stream programming on graphics engines. In Proceedings of the 16th International Conference on Architectural Support for Programming Languages and Operating Systems (ASPLOS XVI). 381392.

Lei Huang, Deepak Eachempati, Marcus W. Hervey, and Barbara Chapman. 2009. Exploiting global optimizations for OpenMP programs in the OpenUH compiler. In Proceedings of the 14th ACM SIGPLAN Symposium on Principles and Practice of Parallel Programming (PPoPP'09). 289-290.

Thomas B. Jablin, James A. Jablin, Prakash Prabhu, Feng Liu, and David I. August. 2012. Dynamically managed data for CPU-GPU architectures. In Proceedings of the 10th International Symposium on Code Generation and Optimization (CGO'12). 165-174.

Thomas B. Jablin, Prakash Prabhu, James A. Jablin, Nick P. Johnson, Stephen R. Beard, and David I. August. 2011. Automatic CPU-GPU communication management and optimization. In Proceedings of the 32 nd ACM SIGPLAN Conference on Programming Language Design and Implementation (PLDI'11). 142-151.

Lars Karlsson. 2009. Blocked in-place transposition with application to storage format conversion. Technical Report UMINF 09.01. Umea University, Umea, Sweden.

Onur Kayiran, Adwait Jog, Mahmut Taylan Kandemir, and Chita Ranjan Das. 2013. Neither more nor less: Optimizing thread-level parallelism for GPGPUs. In Proceedings of the 22nd International Conference on Parallel Architectures and Compilation Techniques (PACT'13). 157-166.

Jungwon Kim, Honggyu Kim, Joo Hwan Lee, and Jaejin Lee. 2011. Achieving a single compute device image in OpenCL for multiple GPUs. In Proceedings of the 16th ACM Symposium on Principles and Practice of Parallel Programming (PPoPP'11). 277-288.

Jaekyu Lee, Nagesh B. Lakshminarayana, Hyesoon Kim, and Richard Vuduc. 2010b. Many-thread aware prefetching mechanisms for GPGPU applications. In Proceedings of the 43rd Annual IEEE/ACM International Symposium on Microarchitecture (MICRO'43). 213-224.

Seyong Lee and Rudolf Eigenmann. 2010. OpenMPC: Extended OpenMP programming and tuning for GPUs. In Proceedings of the ACM IEEE International Conference for High Performance Computing, Networking, Storage, and Analysis (SC'10). 1-11.

Seyong Lee, Seung-Jai Min, and Rudolf Eigenmann. 2009. OpenMP to GPGPU: A compiler framework for automatic translation and optimization. In Proceedings of the 14th ACM SIGPLAN Symposium on Principles and Practice of Parallel Programming (PPoPP'09). 101-110.

Victor W. Lee, Changkyu Kim, Jatin Chhugani, Michael Deisher, Daehyun Kim, Anthony D. Nguyen, Nadathur Satish, Mikhail Smelyanskiy, Srinivas Chennupaty, Per Hammarlund, Ronak Singhal, and Pradeep Dubey. 2010a. Debunking the 100X GPU vs. CPU myth: An evaluation of throughput computing on CPU and GPU. In Proceedings of the 37th Annual International Symposium on Computer Architecture (ISCA'10). 451-460.

LLVM. 2013. The LLVM Compiler Infrastructure Project. Retrieved October 18, 2014, from http://llvm.org/.

John $\mathrm{Lu}$ and Keith D. Cooper. 1997. Register promotion in C programs. In Proceedings of the ACM SIGPLAN Conference on Programming Language Design and Implementation (PLDI'97). 308319 .

Chi-keung Luk, Sunpyo Hong, and Hyesoon Kim. 2009. Qilin: Exploiting parallelism on heterogeneous multiprocessors with adaptive mapping. In Proceedings of the 42nd Annual IEEE/ACM International Symposium on Microarchitecture (MICRO'42). 45-55.

Christos Margiolas and Michael F. P. O’Boyle. 2014. Portable and transparent host-device communication optimization for GPGPU environments. In Proceedings of the Annual IEEE / ACM International Symposium on Code Generation and Optimization (CGO'14). 55.

NVIDIA Corp. 2013. NVIDIA CUDA. Retrieved October 18, 2014, from http://developer.nvidia.com/object/ cuda.html.

William Ogilvie, Pavlos Petoumenos, Zheng Wang, and Hugh Leather. 2014. Fast automatic heuristic construction using active learning. In Proceedings of the Workshop on Languages and Compilers for Parallel Computing (LCPC'14).

Omini Compiler Project. 2009. NAS Parallel Benchmark v2.3 OpenMP C Version. Retrieved October 18, 2014, from http://www.hpcs.cs.tsukuba.ac.jp/omni-compiler/download/download-benchmarks.html.

OpenACC. 2013. The OpenACC Application Program Interface. Retrieved October 18, 2014, from http://www. openacc-standard.org/.

PathScale Inc. 2013. NPB2.3-OpenACC-C. Retrieved October 18, 2014, from https://github.com/pathscale/ NPB2.3-OpenACC-C. 
Portland Group. 2010. PGI Fortran \& C Accelerator Programming Model. White Paper. Retrieved October 18, 2014, from http://www.pgroup.com/lit/whitepapers/pgi_accel_prog_model_1.2.pdf.

J. Ross Quinlan. 1993. C4.5: Programs for Machine Learning. Morgan Kaufmann, San Francisco, CA.

Jagannathan Ramanujam and Ponnuswamy Sadayappan. 1989. A methodology for parallelizing programs for multicomputers and complex memory multiprocessors. In Proceedings of the ACM/IEEE Conference on Supercomputing (Supercomputing'89). 637-646.

Shane Ryoo, Christopher I. Rodrigues, Sara S. Baghsorkhi, Sam S. Stone, David B. Kirk, and Wen-mei W. Hwu. 2008. Optimization principles and application performance evaluation of a multithreaded GPU using CUDA. In Proceedings of the ACM SIGPLAN Symposium on Principles and Practice of Parallel Programming (PPoPP'08). 73-82.

Sangmin Seo, Gangwon Jo, and Jaejin Lee. 2011. Performance characterization of the NAS Parallel Benchmarks in OpenCL. In Proceedings of the IEEE International Symposium on Workload Characterization (IISWC'11). 137-148.

Jaewoong Sim, Aniruddha Dasgupta, Hyesoon Kim, and Richard Vuduc. 2012. A performance analysis framework for identifying potential benefits in GPGPU applications. In Proceedings of the 17th ACM SIGPLAN Symposium on Principles and Practice of Parallel Programming (PPoPP'12). 11-22.

Bjarne Steensgaard. 1996. Points-to analysis in almost linear time. In Proceedings of the 23rd ACM SIGPLAN-SIGACT Symposium on Principles of Programming Languages (POPL'96). 32-41.

John A. Stratton, Vinod Grover, Jaydeep Marathe, Bastiaan Aarts, Mike Murphy, Ziang Hu, and Wen-mei W. Hwu. 2010. Efficient compilation of fine-grained SPMD-threaded programs for multicore CPUs. In Proceedings of the 8th Annual IEEE/ACM International Symposium on Code Generation and Optimization (CGO'10). 111-119.

I-Jui Sung, Juan Gómez-Luna, José María González-Linares, Nicolás Guil, and Wen-Mei W. Hwu. 2014. Inplace transposition of rectangular matrices on accelerators. In Proceedings of the 19th ACM SIGPLAN Symposium on Principles and Practice of Parallel Programming (PPoPP'14). 207-218.

I-Jui Sung, Geng D. Liu, and Wen-Mei W. Hwu. 2012. DL: A data layout transformation system for heterogeneous computing. In Proceedings of Innovative Parallel Computing (InPar). 1-11.

I-Jui Sung, John A. Stratton, and Wen-Mei W. Hwu. 2010. Data layout transformation exploiting memorylevel parallelism in structured grid many-core applications. In Proceedings of the 19th International Conference on Parallel Architectures and Compilation Techniques (PACT'10). 513-522.

Georgios Tournavitis, Zheng Wang, Björn Franke, and Michael O'Boyle. 2009. Towards a holistic approach to auto-parallelization. In Proceedings of the ACM SIGPLAN Conference on Programming Language Design and Implementation (PLDI'09). 177-187.

Sain-Zee Ueng, Melvin Lathara, Sara S. Baghsorkhi, and Wen-Mei W. Hwu. 2008. CUDA-Lite: Reducing GPU programming complexity. In Languages and Compilers for Parallel Computing. Lecture Notes in Computer Science, Vol. 5335. Springer, 1-15.

University of Illinois at Urbana-Champaign (UIUC). 2013. Parboil Benchmark Suite. Retrieved October 18, 2014, from http://impact.crhc.illinois.edu/Parboil/parboil.aspx.

Sven Verdoolaege, Juan Carlos Juega, Albert Cohen, José Ignacio Gómez, Christian Tenllado, and Francky Catthoor. 2013. Polyhedral parallel code generation for CUDA. ACM Transactions on Architecture and Code Optimization 9, 4, Article No. 54.

Zheng Wang and Michael F. P. O’Boyle. 2009. Mapping parallelism to multi-cores: A machine learning based approach. In Proceedings of the 14th ACM SIGPLAN Symposium on Principles and Practice of Parallel Programming (PPoPP'09). 75-84.

Zheng Wang and Michael F. P. O’Boyle. 2010. Partitioning streaming parallelism for multi-cores: A machine learning based approach. In Proceedings of the 19th International Conference on Parallel Architectures and Compilation Techniques (PACT'10). 307-318.

Zheng Wang and Michael F. P. O'Boyle. 2013. Using machine learning to partition streaming programs. ACM Transactions on Architecture and Code Optimization 10, 3, Article No. 20.

Zheng Wang, Daniel Powell, Björn Franke, and Michael F. P. O’Boyle. 2014a. Exploitation of GPUs for the parallelisation of probably parallel legacy code. In Compiler Construction. Lecture Notes in Computer Science, Vol. 8409. Springer, 154-173.

Zheng Wang, Georgios Tournavitis, Björn Franke, and Michael F. P. O’Boyle. 2014b. Integrating profiledriven parallelism detection and machine-learning-based mapping. ACM Transactions on Architecture and Code Optimization 11, 1, Article 2.

Yuan Wen, Zheng Wang, and Michael O’Boyle. 2014. Smart multi-task scheduling for OpenCL programs on CPU/GPU heterogeneous platforms. In Proceedings of the 21st Annual IEEE International Conference on High Performance Computing (HiPC'14). 
Michael Wolfe. 2010. Implementing the PGI accelerator model. In Proceedings of the 3rd Workshop on General-Purpose Computation on Graphics Processing Units (GPGPU'10).

Yi Yang, Ping Xiang, Jingfei Kong, and Huiyang Zhou. 2010. A GPGPU compiler for memory optimization and parallelism management. In Proceedings of the ACM SIGPLAN Conference on Programming Language Design and Implementation (PLDI'10). 86-97.

Received December 2013; revised October 2014; accepted October 2014 